\title{
Challenges for Conducting the Tuberculosis Prevalence Survey and Widening the Scope for Ending TB from Low-Income Countries by 2035
}

\author{
Gokul Mishra $^{1^{*}}$, Dr. Tom Wingfield ${ }^{2}$, Dr. Sharad Kumar Sharma ${ }^{3}$ \\ ${ }^{1}$ PhD in Public Health Student, Texila American University, Guyana \\ ${ }^{2}$ Social Medicine, Infectious Diseases, and Migration (SIM) Group, Department of Global \\ Public Health Sciences, Karolinska Institute, Stockholm, Sweden, Tropical and Infectious \\ Disease Unit, Liverpool University Hospitals NHS Foundation Trust, Liverpool, UK \\ ${ }^{3}$ National Tuberculosis Control Centre, Nayathimi, Bhaktapur Nepal
}

\begin{abstract}
The tuberculosis prevalence survey conducted in 2018-2019 has given the new estimation of TB prevalence $(117,000)$ in Nepal. Out of the estimated burden, about 32,000 people were diagnosed and registered in the National Tuberculosis Programme for treatment in 2019. More than half of the projected tuberculosis cases are still missing in the country, which is a major challenge for achieving the END TB targets by 2035. The study aims to identify the challenges of carrying out the National Tuberculosis Prevalence Survey (NTPS) and recommend possible ways to mitigate the encounters in the future study. All tuberculosis prevalence survey reports were obtained from the World Health Organization as grey literature and analyzed the methods that they used to carry out the survey, challenges they faced while conducting the surveys, and programme implications after the surveys. The studies used a cross-sectional population-based random sampling method to select the clusters using the proportional population to size (PPS) method to select primary and secondary sampling units. Multiple challenges existed to conduct the survey in the country. The logistical and transportation of sputum, equipment, and human resources in different geographical terrain, the low positive rate in culture method and less priority given for health-seeking behavior and TB risk factors in the survey. There are several challenges for conducting a nationwide survey. However, they can be resolved by developing alternatives and communicating the plan in advance. The survey always provides the ground to improve and expand the scope of strategies and interventions, which leads to achieving the desired goals in the programme.
\end{abstract}

Keywords: END Tuberculosis, National Tuberculosis Programme, Probability Population to Size, Tuberculosis, Tuberculosis Prevalence Survey.

\section{Introduction}

Tuberculosis (TB) is a communicable and leading cause of disease, and it is rising as a public health challenge worldwide. Globally, an estimated 10 million people developed TB disease in 2019, of whom approximately 3 million were not diagnosed, treated, or notified to national tuberculosis programs (NTPs). Of the remaining 7 million, many experienced substantial delays in accessing and receiving appropriate diagnosis and care. This unacceptable situation leads to unnecessary disability and loss of life and impedes tuberculosis control because of the onward transmission of TB at both a household and community level. To rectify these shortcomings and eliminate tuberculosis, new strategies are urgently required to find the missing cases, enhance tuberculosis case notification rates, and support people with TB to access quality care and become cured [1]. 
Tuberculosis (TB) remains a public health threat in Nepal, and its responsible for ill-health of thousands of people annually. Nepal had an estimated TB prevalence of 215 TB cases per 100,000 population and an incidence of $151 / 100,000$ in 2019. These estimations based on calculations by the WHO, which are informed by expert opinions, tuberculin skin test surveys, and trends in TB case notification imply that rates of TB have been static in Nepal since 2015. TB also ranks as the leading cause of death in the country in 2019 as well.

There were 32,043 TB cases (all forms of TB) registered at the NTP system in 2018/19, which includes 31,397 incidents of TB cases (new and relapse). Among the registered cases at NTP, there were $37 \%$ female and $63 \%$ male TB patients. The national case notification rate (all forms of TB) was 109 / 100,000 population in 2019 [2].

There are multiple interlinked risk factors associated with TB exposure, infection, and progression to TB disease, which may explain the reasons behind this trend. First, exposure to TB (e.g., the number of viable TB bacilli to which an individual is exposed) and transmission rates are influenced by proximity and duration of exposure to asymptomatic individuals with pulmonary TB and the amount of $\mathrm{TB}$ that individual is producing. Thus, transmission can be affected by the extent of pulmonary TB disease, duration, and frequency of cough, poor cough hygiene or self-isolation measures, overcrowded living circumstances (e.g., due to poverty, incarceration, shared accommodation), occupation (e.g., high-risk jobs such as mining and healthcare), or belonging to an underserved group (e.g., indigenous populations). Second, many other individual-level risk factors influence the likelihood of acquiring TB infection and the development of TB disease. Such risk factors include human immunodeficiency virus (HIV), poverty, malnutrition, chronic renal impairment, diabetes, extremes of age, excess alcohol consumption, smoking, and indoor air pollution. Barriers to accessing TB diagnostic and care services can influence TB rates because diagnostic delay may lead to more severe clinical disease, which in turn increases the likelihood of onward transmission of TB disease in the community [3]. Moreover, stigma and discrimination related to $\mathrm{TB}$ illness, especially in areas with high HIV prevalence, can lead to a reluctance to disclose TB status and seek TB diagnosis and care [4]. Without addressing this individual, household, and health systems-level risk factors for TB disease, TB control strategies, whether at a national or international level, will be unable to eliminate TB.

The study aims to identify the challenges to carry out the National Tuberculosis Prevalence Survey (NTPS) and recommends the possible ways to mitigate the issues for future study and thereby inform national policy and contribute to the National Strategic Plan to eliminate TB in Nepal. It is expected that outputs from the study will include the development of a plan to reduce diagnosis and treatment delays through improved access to care, education to the patients, engagement of informal care providers (like traditional healers), strengthening private health care providers in TB program, and potentially contribute to design of socioeconomic interventions to address the biosocial aspects of TB.

\section{Methods and Materials}

The data were analysed from Nepal National Tuberculosis Prevalence Survey (TBPS) $2018 / 19$, and the systematic review of similar nature of surveys mainly carried out in Asian and Sub-Saharan African Countries after 2000. The survey carried out in Nepal was done at the selected clusters before organizing the TB screening camps. There were 93,085 people enumerated in 99 clusters of 55 districts out of 77 districts of Nepal. Out of 93,085 enumerated, $63 \%(58,956)$ of the people were eligible for the survey, and of them, 54,200 (92\%) invited people attended the survey. The 
participant's people were screened for TB by symptom screening and Chest X-ray methods, and 15,212 (28\%) people were eligible for sputum examination, and $210(1 \%)$ participants not interested to submit sputum. The submitted sputum was examined through X-pert testing 15,010), from Lowenstein-Jensen (LJ) culture method $(7,662)$, and diagnosed prevalent TB cases were 225 .

Researchers also reviewed 28 World Health Organisation (WHO) reports of TBPS from Asia $(n=14)$ and sub-Saharan Africa $(n=14)$ published after 2000. All surveys were population-based and followed WHO guidance, including the probability proportional to size method to determine nationally representative sample size. All surveys used a TB symptom screening questionnaire and chest radiograph to identify people with suspected TB disease and Acid-Fast Bacilli (AFB) smear or fluorescence microscopy to diagnose TB. In all countries except Eritrea, TB diagnosis was supplemented by mycobacterial culture (either LJ or Ogawasolid media), with some countries also using MGIT. After 2011, most surveys also supplemented smear and culture with molecular diagnosis using GeneXpert.

\section{Results}

Nepal faced several challenges to carry out the national wide tuberculosis prevalence survey, different than the Asian and SubSaharan African countries. The challenges were mainly the new federal structure of the country, geographical distribution in different terrain, low positivity rate in culture diagnostic method, inadequate health-seeking behavior-related questions covered, and less considered for the TB disease risk factors aspects in the survey. They are presented in detail in the Table1.

Table1. Challenges Faced during Carrying out National Tuberculosis Prevalence Survey in Nepal

\begin{tabular}{|c|c|c|}
\hline Domain & Challenges/limitations & Possible options for future study \\
\hline \multirow[t]{3}{*}{$\begin{array}{l}\text { New federal } \\
\text { structure }\end{array}$} & $\begin{array}{l}\text { Already defined urban and rural strata were } \\
\text { heavily affected by the new federal structure }\end{array}$ & \multirow[t]{3}{*}{$\begin{array}{l}\text { Development of alternative plan } \\
\text { considering possible challenges }\end{array}$} \\
\hline & $\begin{array}{l}\text { Division of the districts and integrating wards } \\
\text { and village development committee created } \\
\text { difficulties to adjust the population }\end{array}$ & \\
\hline & $\begin{array}{l}\text { Absent of Tuberculosis focal person at the } \\
\text { districts and province }\end{array}$ & \\
\hline Geographical & $\begin{array}{l}\text { Nepal is topographically divided into mountain, } \\
\text { hill and plain and difficult to manage the survey } \\
\text { in hill and mountain especially in the } \\
\text { transportation of logistics, human resources and } \\
\text { sputum to National Reference Laboratory (NRL) }\end{array}$ & $\begin{array}{l}\text { Well and logical plan to be } \\
\text { developed to avoid these kinds of } \\
\text { difficulties }\end{array}$ \\
\hline Diagnostic & $\begin{array}{l}\text { Culture positivity was low, high chances to the } \\
\text { underrepresentation of TB cases }\end{array}$ & $\begin{array}{l}\text { Explore the causes of low positivity } \\
\text { rate in culture method than the } \\
\text { standard and develop the plan to } \\
\text { reduce this problem in the future } \\
\text { study }\end{array}$ \\
\hline \multirow[t]{2}{*}{$\begin{array}{l}\text { Health Seeking } \\
\text { Behavior }\end{array}$} & $\begin{array}{l}\text { Insufficient questions regarding health-seeking } \\
\text { behavior covered and questions related to TB } \\
\text { disease risk factors were missed in the survey }\end{array}$ & \multirow{2}{*}{$\begin{array}{l}\text { Related and sufficient questions } \\
\text { need to be included in the health- } \\
\text { seeking behavior questionnaire and } \\
\text { disease risk factors aspects should } \\
\text { also be collected in the survey }\end{array}$} \\
\hline & $\begin{array}{l}\text { Participants were confused to differentiate } \\
\text { between hospital and health centres }\end{array}$ & \\
\hline
\end{tabular}


Various opportunities have been created and the scope to strengthen the program has been widened from the tuberculosis prevalence survey, which would work as a cornerstone to achieve the targets of the END TB strategy in Nepal. The programme has the opportunity to develop and implement the strategies to achieve the targets of End TB in the country.

1. Fostering political commitment, partnerships beyond health, engagement of all levels, networking with national and international stakeholders to increase investment and sustain TB program in the country.

2. Reach the unreached with high-quality and patient-friendly tuberculosis services under the framework of universal health coverage (UHC).

3. Strengthening the current diagnostic system and improve the accessibility of highly sensitive TB diagnostic services to finding out missing tuberculosis cases in high-risk populations.

4. Enhancing the existing drug supply system and increase accessibility of effective tuberculosis treatment management in the community.

5. Promote community TB care and management, enhancing the capacity of local stakeholders.

6. Mapping ethnic communities, especially Dalits and Janjati habited areas, develop and implement focus programs to finding out the missing cases.

7. Rebuilt the program structure and network, considering the federal system of the country to implement strategy effectively.

8. Advancing the research and documentation capacities on TB diagnosis, treatment, care and support.

9. Wider and meaningful engagement of private health care providers in $\mathrm{TB}$ elimination initiatives.

\section{Discussion}

Nepal National Tuberculosis Programme (NTP) successfully conducted the tuberculosis prevalence survey despite several challenges in the country. The challenges that were encountered included the new structure of the country, typical geographical distributions (Mountain, Hill, and Flat) and difficult to transport the logistics, human resources, and sputum to National Reference Laboratories (NRL), low TB positivity rate in the culture method, insufficient questions covered under the health-seeking behavior and ignore the TB disease risk factor's part. The systematic reviews of TBPS carried out in 28 countries (14 Asian and 14 Sub-Saharan African) after 2000 demonstrate the challenges of conducting a nationwide tuberculosis prevalence survey (TBPS) is varies from country to country depending upon its nature and specialty. There were some common limitations to carry out the survey; geographical difficulties in several countries, low participation in urban areas, especially the young male population, long and complicated procurement process for logistics, the prevalence of tuberculosis in under-five children, pulmonary clinical diagnosis (PCD) and Extra Pulmonary (EP) were not assessed and results extrapolating from routine surveillance data, difficult to manage chest Xray (CXR) machine (including broken down) in the field, delayed in releasing domestic funding for the survey, a natural disaster like flooding and landslide and security challenges, low sensitive diagnostic tool (microscope) was used to diagnose TB, high turnover of staff in the field, assessed TB risk factors (diabetic, HIV) based on self-reported by the participants instead of testing their blood, participants could not recall their past TB treatment history due to fear of stigma in Asian surveyed countries [5, 6, $15-18,7-14]$ 
The African TBPS conducted countries experience the prevalence of $\mathrm{TB}$ in under-five children, PCD and EP were not assessed and resulted extrapolating from routine surveillance data, the high workload in the lab due to use of the microscope and high contamination rate in sputum culture, low participation in urban areas especially young male population, Long and complicated bureaucratic procurement process for logistics, the survey only provided a national estimate. It did not give sub-national and below levels, a natural disaster like flooding and landslide and security challenges, difficult to transport the sputum from remote and rural areas, poor internet connectivity in the filed resulting delay in sending CXR and data on time [19, 20, 29-32, 21-28].

Despite the various challenges to carry out nationwide surveys, wonderful opportunities have been created in the history of NTP to widen its scope to eliminate the tuberculosis disease from the country. Increase political commitments, engage all levels of health care providers, strengthen the partnership with several concerned stakeholders, development and implementation of innovative approach to reach and unreached population, increase the accessibility of advance and sensitive diagnostic tools and techniques, promote community meaningful engagement of TB survivors, community-based organization and empower communities to fight against tuberculosis, establish a robust mechanism to assess the TB disease risk factors to break the chain of disease transmission at the individual, household, community, and health system levels. Besides this important research would also a good prospect to garner evidence-based information and develop sound strategies and interventions in the programme.

The surveys carried out across the world also provided a similar landscape to strengthen their programme in the country. They are;

1. Increase accessibility of TB diagnostic facilities with sensitive and advance technology, including chest radiography with a clear algorithm.

2. Develop and implement the innovative and focus interventions to the high risk and targeted communities to mitigate the prevalence notification (PN) gaps.

3. Ensure a high level of political commitment to increase the domestic investment to implement the robust and intensive program in the country.

4. Widen the partnerships with intersectoral government, private health care providers, civil society organization, communities, national and international partners, and donors.

5. Promote digital, case, and web-based recording and reporting system in the program.

6. Design and implement the program to address the TB disease risk factors at the individual, household, community, and health system levels.

\section{Conclusion}

There are multiple challenges to conducting TPS that are some logistical obstacles, topography and natural disasters, error, and limitations in molecular diagnostic test results, less attention to incorporate health-seeking behaviour and TB disease risk factors related questions in the survey. Countries intending to conduct TPBS should focus on potential modifiable challenges during the TBPS planning process. These include developing an alternative plan considering geographical disparities, envisioned potential errors before starting sputum examination, and bearing in mind that the seeking behaviours and $\mathrm{TB}$ disease risk factors are also significant contributors and need to be included in the survey designing.

The surveys also provided a platform to the high TB burden countries with low-income countries to increase political commitment to increase domestic investment, engage all care providers, and secure meaningful partnership, 
mobilization and empowerment to the TB survivors, communities, and community-based organization and local community actors to manage tuberculosis programme in the country. There is an opportunity to increase access and expend sensitive diagnostic technology, including chest X-ray, development, and implement evidence-based interventions and planning to reach the unreached across the world, which would be a milestone to eliminate tuberculosis from the world and contribute to making TB free world.

\section{References}

[1] World Health Organisation, (2019), Global Tuberculosis Report.

[2] Ministry of Health and Population, National Tuberculosis Control Centre, (2020), Annual Progress Report.

[3] Onuka O, et al., 2018, Effectiveness of Contact Tracing of Index Tuberculosis Cases in Nigeria. Adv Infect Dis. 2018;08(04):173-99.

[4] Amo-Adjei J, 2016., Individual, household and community level factors associated with keeping tuberculosis status secret in Ghana. BMC Public Health [Internet]. 2016;16(1):1-9 http://dx.doi.org/10.1186/s12889-016-3842.

[5] Ministry for Health (2016), Report of the first national tuberculosis prevalence survey in Mongolia 2014-2015.

[6] Ministry of Health and Sports (2019), 4 th National TB Prevalence Survey 2017/2018, Myanmar, short version, version 1 as of Aug 2019.

[7] Ministry of Health and Family Welfare (2016), National Tuberculosis Prevalence Survey Bangladesh 2015-2016.

[8] Ministry of Health (2015), Indonesia Tuberculosis Prevalence Survey Indonesia 2013-14.

[9] Floyd K, et.al, Prevalence survey update. Available from:

http://www.stoptb.org/countries/tbteam/assets/docu ments/MeetingDocs6/12PrevalenceSurvey_EpiAsse ssments_TBsurveillanceassessments.pdf.

[10] King NR, et.al, 2006, Kingdom of Cambodia Ministry of Health Report of. 2006;(September).

\section{Acknowledgments}

The authors would like to thank the Director of the National Tuberculosis Control Centre (NTCC) for his approval, guidance, and support to prepare this manuscript. Finally, we would also like to thank all the contributors and supporters to complete this work successfully.

\section{Conflict of Interest}

The authors declare that there is no conflict of interest.

[11]Ministry of Health (2012), report second Tuberculosis N, Program C. Report Second National Tuberculosis Prevalence Survey, 2011.

[12] Ministry of National Health Services Regulation and Services (2010/11), Prevalence of Pulmonary Tuberculosis among Adult Population of Pakistan.

[13]Ministry of Health (2017), National Tuberculosis Prevalence Survey 2016 Philippines.

[14]Disease Control Bureau of the Ministry of Health (2010), Report on the 5th National Tuberculosis Epidemiological Survey in China.

[15]Ministry of Health (2006), determining the Tuberculosis Burden in Eritrea-new methodological approach, 2005.

[16] World Health Organisation, Country Office Report (2009), Report of the Bangladesh Nationwide Tuberculosis Disease-cum -infection prevalence survey (2007-2009).

[17]Ministry of Health, Vietnam National Tuberculosis Programme (2008), Draft Report National Tb Prevalence Survey 2006-7.

[18]Ministry of Health (2005), Indonesia Tuberculosis Prevalence Survey in Indonesia 2004.

[19] Kapata N, et al. (2016), The Prevalence of Tuberculosis in Zambia: Results from the First National TB Prevalence 2013-4.

[20] Federal Democratic Republic of Ethiopia, Ministry of Health (2011), First Ethiopian National Population Based Tuberculosis Prevalence Survey, Ababa A.

[21]Ministry of Health (2018), Kenya National Tuberculosis Prevalence Survey. 
[22] Ministry of Health (2006), determining the Tuberculosis Burden in Eritrea-new methodological approach, 2005.

[23] Republic of Zimbabwe Ministry of Health and Child Care (2014), the Zimbabwe National Population based Tuberculosis Prevalence Survey Report.

[24] Ministry of Health and Social Welfare (2014), the Gambian Survey of Tuberculosis Prevalence (GAMSTEP).

[25] The United Republic of Tanzania, Ministry of Health and Social Welfare (2013), the first National Tuberculosis Prevalence Survey in the United Republic of Tanzania final report;32(C):120.

[26] Bradshaw MJ, et, al. (2020), Neurologic Manifestations of Systemic Rheumatologic Diseases. Current Clinical Neurology. p. 321-42.
[27] Ministry of Health and Social Services (2019), Namibia Tuberculosis Disease Prevalence Survey report.

[28] Government of the Republic of Zambia, Ministry of Health (2013-14), National Tuberculosis Prevalence Survey Report- technical report.

[29]Federal Republic of Nigeria (2012), Report of the First National TB Prevalence Survey in Nigeria. Available from: http://ntblcp.gov.ng.

[30] Republic of Rwanda, Ministry of Health (2014), Report of the first National Pulmonary Tuberculosis Prevalence Survey in Rwanda;15-98.

[31] The Republic of Uganda, Ministry of Health (2014-15), the Uganda National Tuberculosis Prevalence Survey, 2014-2015;2014-5.

[32] Federal Ministry of Health (2013-14), Report on National Tuberculosis Prevalence Survey, Sudan. 